\title{
Iterative Detection Aided H.264 Wireless Video Telephony Using Irregular Convolutional Codes
}

\author{
Nasruminallah, R. G. Maunder and L. Hanzo \\ School of ECS, University of Southampton, SO17 1BJ, UK. \\ http: //www-mobile.ecs.soton.ac.uk, \\ Email: $\{1 \mathrm{~h}\} @ e c s$. soton.ac.uk
}

\begin{abstract}
In this paper we evaluate the performance of a DataPartitioned (DP) H.264 coded video transmission system using Unequal Error Protection (UEP) IrRegular Convolutional Codes (IRCC). Using UEP, perceptually more important bits are provided with more strong protection relative to less important bits. An iterative detection aided combination of IRCC and a rate- 1 precoder was used to improve the overall BER performance and to enhance the objective video quality expressed in terms of Peak Signal-to-noise Ratio (PSNR). The effect of different error protection schemes on the attainable system performance is demonstrated, while keeping the overall bit-rate budget constant for the transmission of DP H.264 source coded video over correlated narrowband Rayleigh fading channels. In this paper we exploited the high design flexibility of IRCCs, which constitutes a family of different rate subcodes, while maintaining an excellent iterative decoding convergence performance. Additionally, due to the use of different-rate subcodes, IRCCs have the capability of providing UEP for the H.264 coded video stream. An EXIT chart matching procedure was used for the design of our specific IRCC. Additionally, EXIT charts were used for analysing the attainable system performance of various error protection schemes employed. Explicitly, our experimental results show that the proposed UEP scheme using IRCC outperforms its Equal Error Protection (EEP) counterpart employing regular convolutional codes by about $0.5 \mathrm{~dB}$ $E_{b} / N_{0}$ at the PSNR degradation point of $1 d B$.
\end{abstract}

\section{Motivation AND BACKGROUND}

Multimedia source coded information, such as speech, audio and video typically exhibit unequal sensitivity to channel errors [1,2]. Therefore, Unequal Error Protection (UEP) is used by exploiting the innate UEP capability of IrRegular Convolutional Codes (IRCCs) due to the employment of various constituent codes having different coding rates to ensure that the perceptually more important bits suffer from a lower number of channel errors. The construction of IRCCs was studied by Tuchler and and Hagenauer [3], where a family of different convolutional codes having various coderates were designed with the aid of EXIT charts for achieving an improved iterative decoding behaviour for a serially concatenated system. For wireless multimedia applications maintaining both a high compression efficiency as well as a high integrity, while communicating over heterogeneous communication networks is of primary importance. Therefore, to evaluate the performance of our proposed error protection schemes, we used the high-compression efficient and network-friendly H.264/AVC source codec [4], which employs various variable length coding and predictive coding techniques to achieve compression efficiency. However, these coding techniques make the compressed bit-stream more vulnerable to the effects of channel errors. Due to variable length coding a single bit error in the video stream may render the correct decoding of future code words impossible. Moreover, due to predictive coding the effects of channel errors are likely to be propagated to the neighbouring video blocks. Therefore, various error resilient techniques, such as Data Partitioning DP have been incorporated into the H.264/AVC codec in order to mitigate this error sensitivity problem. Additionally, various error resilient schemes have been proposed in [5], but the price paid is the reduced compression efficiency and increased computational complexity. A serial concatenated code based iterative joint sourcechannel decoding procedure was presented in [6]. Moreover, a irregular variable length coding (IrVLC) scheme designed for near-capacity joint source and channel coding was presented in [7]. Likewise, [8] advocates the employment of state-of-the-art system design principles and the performance of burst-by-burst adaptive transceivers designed for interactive cellular and cordless video telephony. Instead of the traditional serial concatenation of the classic Variable Length Codes (VLC) with a channel code, a parallel concatenated coding scheme was presented in [9] where the VLCs were combined with a turbo code. A joint video and channel coding system employing an iteratively decoded serial concatenation of a Vector Quantisation (VQ) based video codec and a Trellis-Coded Modulation (TCM) scheme was proposed in [7]. The attainable performance improvements of Iterative Source Channel Decoding (ISCD) Scheme employing a specific bit-to-symbol mapping scheme were analysed in [10], in the context of H.264 encoded video signals transmission using UEP and Sphere Packing (SP) modulation aided Differential Space Time Spreading (DSTS) in a non-coherently detected, diversity-aided multiuser scenario. Furthermore, the performance analysis of diverse low-complexity Short Block Codes (SBCs) for the transmission of H.264 coded video stream over correlated narrowband Rayleigh fading channels was presented in [11].

Against this background, the novel contribution of our work is the UEP of DP-aided and H.264/AVC coded stream using EXIT chart-optimised IRCCs. More explicitly, we proposed a serially concatenated turbo transiver, consisting of an iterative combination of an outer IRCC and an inner rate-1 precoder.

The rest of the paper is organised as follows. An overview of H.264 Data Partitioning is provided in Section II. In Section III we portray our system model. The IRCC design strategy is outlined in Section IV, followed by an IRCC design example applied to our proposed system in Section V. Section VI provides the Extrinsic Information Transfer Chart (EXIT) analysis of the proposed error protection schemes. The performance of the proposed system is characterised with the aid of our simulation results in Section VII. Finally, we offer our conclusions in Section VIII.

\section{H.264 DATA PARTITIONING}

The H.264 DP scheme is designed to achieve an enhanced error resilience. Instead of the classic approach of coding all video parameters of a Macro-Block (MB) into a single bit-string representing a video-slice, the H.264 DP may generate three bit-strings per slice referred to as partitions, and hosting different-sensitivity parameters. This provides us with the ability to protect the different sensitivity classes based on their relative importance. The three different partitions generated by the DP mode of H.264 are,

- Type A: It contains the slice header information, motion vectors, quantisation parameters and MB types. When a type A partition is corrupted, the bits of the entire slice are dropped. In this 
case the slice is marked as corrupted and the decoder will apply error concealment techniques using the previous decoded video segments.

- Type B: It carries both the intra-frame coded MB coefficients and the intra-MB coded Block Pattern (CBP) bits, representing blocks within a MB containing non-zero transform coded coefficients.

- Type C: It typically contains inter-frame Motion Compensated Error Residual (MCER) bits and inter-frame CBP bits for MBs encoded using inter-frame compensated prediction, as well as intra-frame CBP and intra-frame MCER bits for the MBs coded using the H.264 specific intra-frame prediction mode.

Therefore, in the H.264 video stream, partition A contains very important information. Similarly, the information contained in partition $\mathrm{B}$ and $\mathrm{C}$ has its own significance in terms of effect on video quality. If partition $\mathrm{B}$ of a slice is present along with $\mathrm{A}$, the intra-frame $\mathrm{MB}$ update is added to the reconstructed frame, while if partition $\mathrm{C}$ is present, the MCER of a slice is reconstructed and added to the motion compensated slice. Recovery from inter-frame error propagation can only be achieved by encoding certain image regions in intra-frame mode by switching off inter-frame prediction for certain MBs, when no feedback channel is available. Typically a limited fraction of the MBs is encoded in the intra-frame coding mode, so that partition B typically hosts the lowest number of bits in an encoded slice.

\section{System OVERVIEW}

The schematic of the proposed videophone arrangement is shown in Figure 1. At the transmitter side, the video sequence is compressed using the H.264/AVC video codec. Then, the output bit-stream representing a video frame consisting of $K$ source coded bits $x_{k}, k=$ $1,2, \ldots K$, is de-multiplexed into three different bit-streams, namely Stream A, Stream B and Stream C, containing the sequentially concatenated partitions of type $\mathrm{A}, \mathrm{B}$ and $\mathrm{C}$ of all the slices per frame, respectively. The de-multiplexers binary output sequences $x_{a}$, $x_{b}$, and $x_{c}$, where we have $a=1,2, \ldots A, b=1,2, \ldots B$ and $c=1,2, \ldots C$, and $K=A+B+C$, are then concatenated according to a specific order, depending on the choice of the error protection scheme applied to the bit-string $x_{i}$. Subsequently, the concatenated bit-string is coded using an IRCC having a specific code rate into the coded string $x_{i}^{\prime}$. The coded string $x_{i}^{\prime}$ is then interleaved using the bitinterleaver $\Pi$ of Figure 1, into the interleaved sequence $\bar{x}_{i}$, which is then encoded by the rate- 1 precoder, characterised by the generator polynomial of $1 /(1+\mathrm{D})[12]$, before transmission. In the iterative decoder of Figure 1, the extent of the statistical independence of the extrinsic information provided by an interleaver is always related to its length [13]. Therefore instead of independently performing the iterative decoding operation on the various frame slices, we integrated all the bits generated by the MBs of the slices of each partition within a given video frame. The integrated bit-strings of each partition are then subsequently concatenated into a single string, which results in a longer interleaver and hence improves the attainable iterative decoding performance without extending the video delay. The precoded bit-stream $y_{i}$ is QPSK modulated and transmitted over a temporally correlated narrowband Rayleigh fading channel, associated with the normalised Doppler frequency of $f_{d}=f_{D} T_{s}=0.01$, where $f_{D}$ is the Doppler frequency and $T_{s}$ is the symbol duration. At the receiver the soft-information obtained after QPSK demodulation is forwarded to the inner decoder. The extracted extrinsic information is then exchanged between the inner and outer decoder, in order to attain the lowest possible BER [14]. At the receiver the soft-information obtained after QPSK demodulation in the form of its Log-Likelihood
Ratio (LLR) representation $D_{M, a}$ is forwarded to the rate- 1 precoder. The rate- 1 precoder's decoder processes this input information and the fed back a priori information $L_{M, a}$ from the outer decoder in order to generate the a posteriori LLR values $L_{M, p}$. The rate1 precoder's a priori LLR values $L_{M, a}$ are subtracted from its a posteriori $\mathrm{LLR}$ values $L_{M, p}$ to generate the extrinsic LLR values $L_{M, e}$, which are subsequently deinterleaved by the soft-bit interleaver of Figure 1, yielding $L_{D, a}$. Then, the soft bits $L_{D, a}$ are passed to the convolutional decoder, which uses the Log MAP algorithm of [15] to compute the a posteriori LLR values $L_{D, p}$. Observe in Figure 1 that $L_{M, a}$ is the interleaved version of $L_{D, e}$, which in turn is generated by subtracting the a prior $i$ information $L_{D, a}$ from the a posterior $i$ information $L_{D, p}$. During iterative decoding the precoder's decoder exploits the a priori information for the sake of providing improved a posterior $i$ LLR values for the outer channel decoder, which in turn exploits the input LLR values for the sake of providing improved a priori information for the precoder in the subsequent iteration. Further details about iterative decoding are provided in [16].

\section{Irregular Convolutional Code Design}

The details of IRCC design can be found in [3]. More explicitly an IRCC consisting of ' $I_{n}^{\prime}$ constituent subcodes is constructed by first selecting a convolutional mother code $C_{1}$ with rate- $r_{1}$. The remaining $\left[I_{n}-1\right]$ subcodes $C_{i}$ of rate- $r_{i}>r_{1}$, where we have $i=2 \cdots I_{n}$, are obtained by puncturing, while subcodes $C_{i}$ with rate- $r_{i}<r_{1}$ are created by adding more generators and by puncturing. In IRCCs $K$ input bits are encoded into $N$ coded bits, where each rate- $r_{i}$ subcode encodes only a fraction $\left(\alpha_{i} r_{i} N\right)$ of information bits and generates $\left(\alpha_{i} N\right)$ encoded bits, with $\alpha_{i}$ representing the relative size of a fraction. The weighting coefficients $\alpha_{i}, i=1 \ldots I_{n}$ and the target code rate of $R \in\left[\begin{array}{ll}0 & 1\end{array}\right]$ should satisfy,

$$
\begin{gathered}
\sum_{i=1}^{I_{n}} \alpha_{i}=1, \text { and } \sum_{i=1}^{I_{n}} \alpha_{i} r_{i}=R, \text { where } \alpha_{i} \in\left[\begin{array}{ll}
0 & 1
\end{array}\right] . \\
\text { V. DESIGN EXAMPLE }
\end{gathered}
$$

In our design example we used a fixed overall coding rate of $R=0.7$, which was assigned to the outer code, because we used a unit rate code as our inner code. As a benchmarker we used a half-rate memory-4 Recursive Systematic Convolutional Code (RSC) as our outer code defined by the generator polynomial $\left(1, g_{1} / g_{0}\right)$, where $g_{0}=1+D+D^{4}$ and $g_{1}=1+D^{2}+D^{3}+D^{4}$ are the feedback and feedforward polynomials, respectively. Then the overall RSC code rate of 0.7 was obtained by puncturing. Alternatively, for the IRCC design, we used the proposed $I_{n}=17$ constituent subcodes

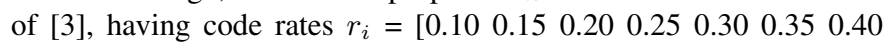

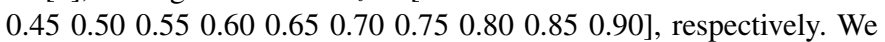
employed the EXIT chart matching algorithm of [17] and selected the target inner code transfer function recorded at $E_{b} / N_{0}=3 \mathrm{~dB}$, which resulted in the weighting vector of $\alpha_{i}=[0.0031120 .029389$ $\left.\begin{array}{llllllllllllllll}0 & 0 & 0 & 0 & 0 & 0 & 0 & 0 & 0 & 0.291014 & 0.657845 & 0 & 0 & 0.0477998 & 0\end{array}\right]$ for the outer IRCC code. By concatenating the three resultant partitions per video frame into a specific order depending on the error protection scheme applied, we can control their relative error protection with the aid of IRCC coding, which provides a stronger error protection for the concatenated bit-stream portion coded by lower-rate subcodes relative to the bit-stream portion coded by the higher-rate subcodes.

\section{EXIT CHART ANALYSIS}

The EXIT [18] function of both the IRCC and benchmarker RSC are shown in Figure 2. In addition to the beneficial UEP capability of the IRCC, it can be observed from the EXIT curves 


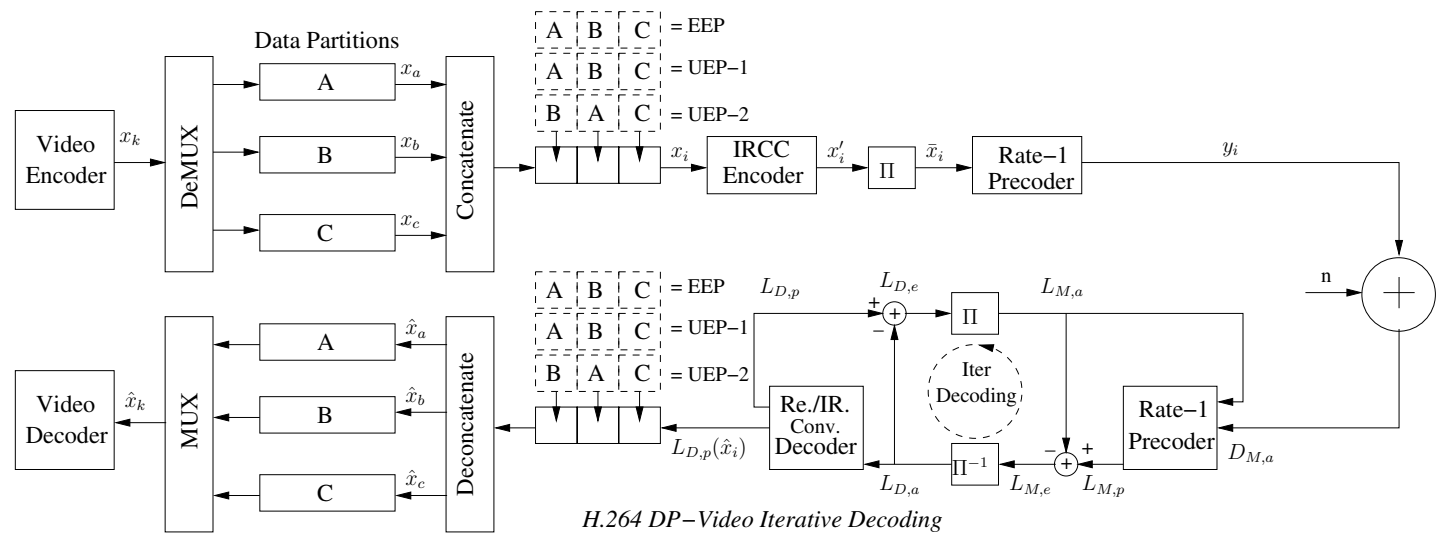

Fig. 1. The proposed system model.

TABLE I

CODE RATES FOR DIFFERENT ERROR PROTECTION SCHEMES

\begin{tabular}{|l|c|c|c|c|}
\hline \multirow{2}{*}{$\begin{array}{l}\text { Protection } \\
\text { Scheme }\end{array}$} & \multirow{2}{*}{$\begin{array}{c}\text { Concatenation } \\
\text { order }\end{array}$} & \multicolumn{3}{|c|}{ Code Rate } \\
\cline { 3 - 5 } & & Outer Code & Inner Code & Overall \\
\hline \hline EEP & {$[$ A-B-C] } & RSC & Rate-1 Precoder & 0.7 \\
UEP-1 & {$[$ A-B-C] } & IRCC & Rate-1 Precoder & 0.7 \\
UEP-2 & {$[$ B-A-C] } & IRCC & Rate-1 Precoder & 0.7 \\
\hline
\end{tabular}

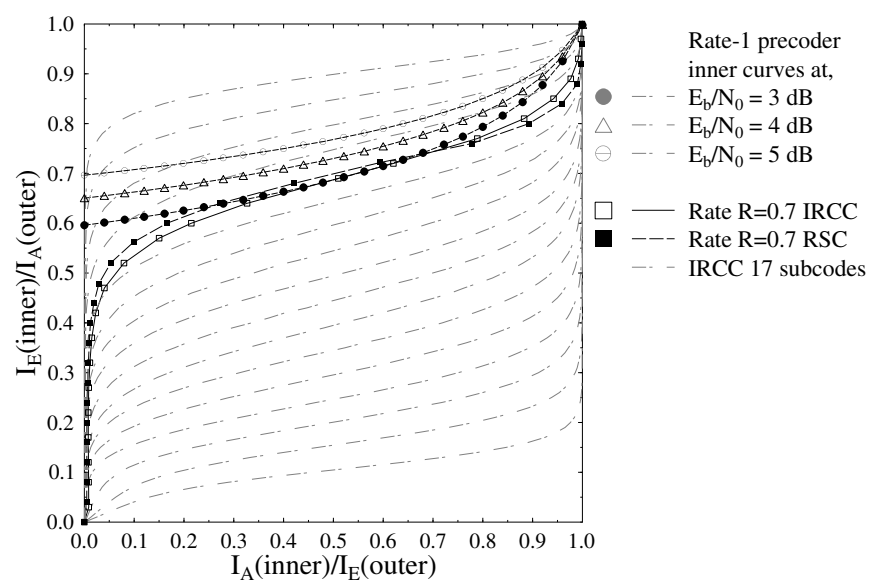

Fig. 2. The EXIT functions of 17 subcodes used in [17] along with rate-1 inner precoder curves and the employed regular/irregular outer code curves.

that there is a wider EXIT chart tunnel between the rate-1 inner precoder's curve and the outer irregular channel code's curve relative to the benchmarker scheme dispensing with the regular outer IRCC channel code. Therefore, given a limited number of affordable iterations, a relatively short interleaver and a wider EXIT tunnel, our designed 0.7-rate IRCC may be expected to perform better than the benchmarker scheme using the identical-rate RSC code. The actual decoding trajectories of the various error protection schemes employing the regular/irregular outer channel codes as well as using the rate- 1 inner precoder detailed in Table I was recorded at $E_{b} / N_{0}=3 d B, 4 d B$ and $5 d B$, as portrayed in Figures 3, 4 and 5 , respectively, where $\mathrm{I}_{\mathrm{A}}$ is the mutual information between the coded bits and the corresponding a priori LLR values input to the decoder, while $\mathrm{I}_{\mathrm{E}}$ is the mutual information between the coded bits and the corresponding a posteriori LLR values output from the decoder. These trajectories were plotted by acquiring the mutual information between the hypothesized and soft-bit values at the input and output of both the inner and outer decoder during the bit-by-bit Monte-Carlo simulation of the iterative decoding algorithm. It may be inferred from the EXIT trajectories of Figures 3, 4 and 5 that as expected, the convergence behaviour of the iterative decoding scheme was improved by the irregular outer channel codes, which provide a wider EXIT tunnel relative to the regular outer channel code, although they have the same code rates.

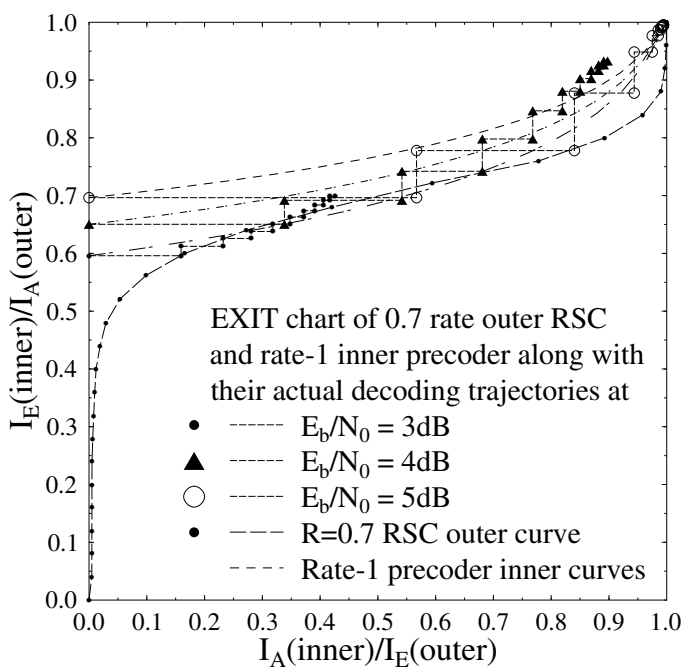

Fig. 3. The EXIT chart and decoding trajectories of the EEP scheme.

\section{System PERformance RESUlts}

The performance results of our proposed system are presented in this section. We used a 45 frame "Akiyo" video sequence [5] in (176x144)-pixel Quarter Common Intermediate Format (QCIF) as our test sequence. This test sequence was encoded using the H.264/AVC JM 13.2 reference video codec at 15 frames-per-second ( $f p s$ ) operated at the target bitrate of $64 \mathrm{kbps}$. Each QCIF frame was partitioned into 9 slices and each slice was composed of 11 MBs. The resultant video encoded clip consists of an intra-coded 'I' frame followed by 44 predicted ' $\mathrm{P}$ ' frames, corresponding to a 3 seconds time-lag between two consecutive 'I' frames at $15 \mathrm{fps}$, in order to reduce inter-frame error propagation. Additionally, we incorporated intra-frame coded MB updates of three randomly dispersed MBs per frame in order to mitigate the effects of error propagation. The insertion of ' $\mathrm{B}$ ' pictures results in an unacceptable loss of lip-synchronisation as a result of the corresponding delay incurred due to the bi-directionally predicted video coding operations [4] and hence was avoided. The remaining system parameters of our experimental setup are listed in Table II. With an interactive videophone senario in mind, the error resilient encoding techniques, such as Flexible Macro-block Ordering (FMO) [4] and the employment of multiple reference frames for interframe motion compensation were turned off, because despite their 
TABLE II

SYSTEMS PARAMETERS

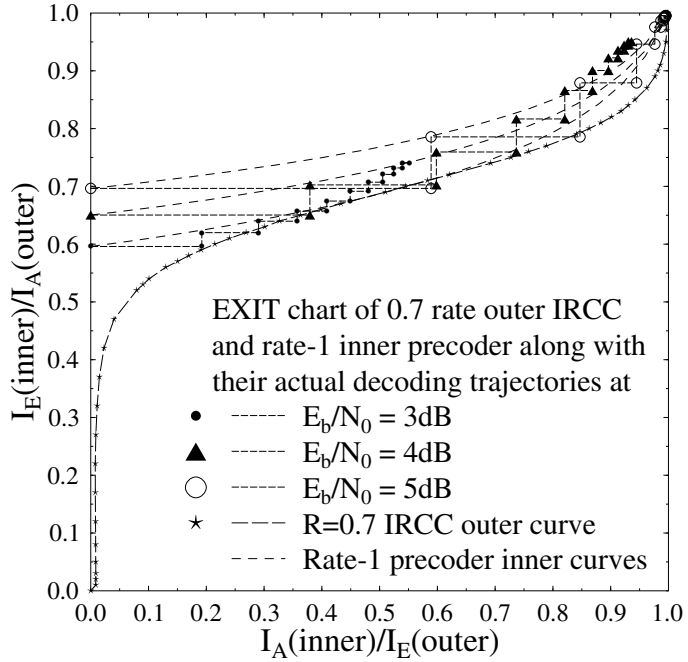

Fig. 4. The EXIT chart and decoding trajectories of the UEP-1 scheme.

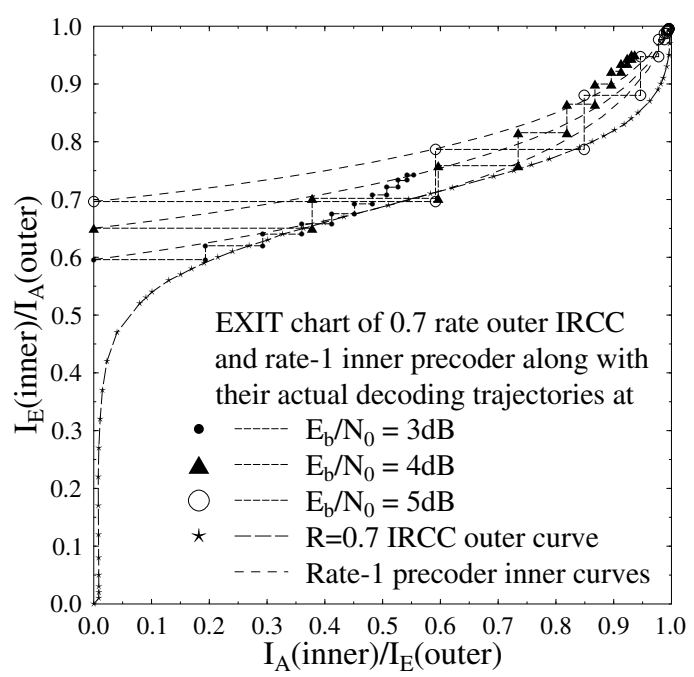

Fig. 5. The EXIT chart and decoding trajectories of the UEP-2 scheme.

substantially increased complexity they typically result in modest video performance improvements in low-motion head-and-shoulders video sequences, such as the "Akiyo" clip [4]. Additionally, only the immediately preceding frame was used for motion search, which results in a reduced computational complexity compared to using multiple reference frames and error concealment was performed using the motion vector recovery algorithm of [4]. Moreover, for the sake of reducing the computational complexity imposed, we limited the number of iterations between the outer code and rate- 1 inner precoder to $I_{t}=10$. For the sake of increasing the confidence in our results, we repeated each 45 -frame experiment 160 times and averaged the generated results. The performance of the system was evaluated by keeping the same overall code rate as well as video rate for both the Equal Error Protection (EEP) and UEP coding schemes. In the UEP-1 scheme of Table I based on the specific concatenation order [A-B$\mathrm{C}$ ] of partition $\mathrm{A}$, followed by partition $\mathrm{B}$ and then $\mathrm{C}$, the highest error protection was provided for partition $\mathrm{A}$, while partition $\mathrm{B}$ was more strongly protected than C. By contrast, the UEP-2 partitions arrangement [B-A-C] of Table I represents another error protection scheme, in which the highest level of error protection is provided for partition $\mathrm{B}$, followed by partition $\mathrm{A}$ and $\mathrm{C}$.

The rationale of the UEP-2 arrangement in comparison to the UEP-

\begin{tabular}{|l|r|}
\hline System Parameters & Value \\
\hline \hline Channel Coding & Re./IR. conv. code \\
Over-all Code Rate & 0.7 \\
Modulation Scheme & QPSK \\
Number of Transmitters, $N_{t}$ & 1 \\
Number of Receivers, $N_{r}$ & 1 \\
Channel & Correlated Rayleigh Fading \\
Normalised Doppler Frequency & 0.01 \\
Interleaver Length & H.264/AVC \\
Source Coding & 64 \\
Bit Rate (Kbps) & 15 \\
Frame Rate (fps) & 9 \\
No of Slices/frame & 11 \\
No of MB's/Slice & 3 \\
Intra-frame MB update/frame & \\
\hline
\end{tabular}

1 scheme, where partition A had the strongest protection is that in low-motion video sequences the slices corrupted due to the loss of partition A are concealed using the motion vector recovery algorithm of [4], which results in less annoying artefacts due to their high correlation across the adjacent frames. However, the loss of partition B along with a correctly received partition A of a particular slice implies that the MV and header information received in partition A will be used to decode the MBs of this slice, encoded either in intra- or inter-frame prediction mode, while the all-important MBs of partition $\mathrm{B}$ containing intra-frame coded $\mathrm{MB}$ coefficients and intraMB CBP bits were completely lost. Additionally, when the intraframe coded MB updates are corrupted, this results in avalanchelike error propagation to the future video frames. Therefore, in low-motion video sequences partition $\mathrm{B}$ is also very important and hence its corruption results in noticeable PSNR degradation of the decoded video sequence. Hence, by providing more error protection to partition $\mathrm{B}$, the correct reception of partition $\mathrm{B}$ is guaranteed for every correctly received partition $\mathrm{A}$, which avoid error propagation to the future video frames due to predictive video coding. Furthermore, typically partition B has the lowest number of bits, therefore the employment of a strong subcode does not result in a significant increase of the overall rate. As a result, using the remaining fraction of the bit-rate budget, a reasonable protection can still be provided for partitions $\mathrm{A}$ and $\mathrm{C}$.

Figure 6 presents the attainable $B E R$ performance of partitions A, $B$ and $C$ when using the EEP, UEP-1 and UEP-2 schemes of Table I, while employing regular/irregular outer channel codes. Naturally, the three partitions of the EEP scheme experience a similar BER, since they are protected equally with the same code rate. In the UEP-1 scheme the partitions $\mathrm{B}$ and $\mathrm{C}$ have a higher BER than partition A, which is due to their higher code rate relative to partiton A. Likewise, partition A of the UEP-2 scheme of Table I has a higher BER relative to partition $\mathrm{B}$, which in turn has a lower BER than partition $\mathrm{C}$.

The performance trends expressed in terms of the $P S N R$ versus $E_{b} / N_{0}$ curves are portrayed in Figures 7. It may be observed in Figure 7 that the UEP-2 scheme provides the best $P S N R$ performance among the three different error protection schemes of Table I across the entire $E_{b} / N_{0}$ region considered. From the simulation results of Figure 7 it is observed that the UEP-1 scheme of Table I results in the worst $P S N R$ performace, when employing of our 0.7-rate IRCC in conjunction with the rate- 1 inner precoder. By contrast, an $E_{b} / N_{0}$ gain of $1 \mathrm{~dB}$ is attained using UEP-2 relative to UEP-1 at the PSNR degradation point of $1 \mathrm{~dB}$. Addtitionally, using the UEP-2 scheme of Table I, an $E_{b} / N_{0}$ gain of about $0.5 \mathrm{~dB}$ may be achieved over the identical-rate EEP benchmarker scheme using the regular RSC channel code of rate 0.7 . Finally, the subjective video quality achieved by the proposed EEP employing regular convolutional code 


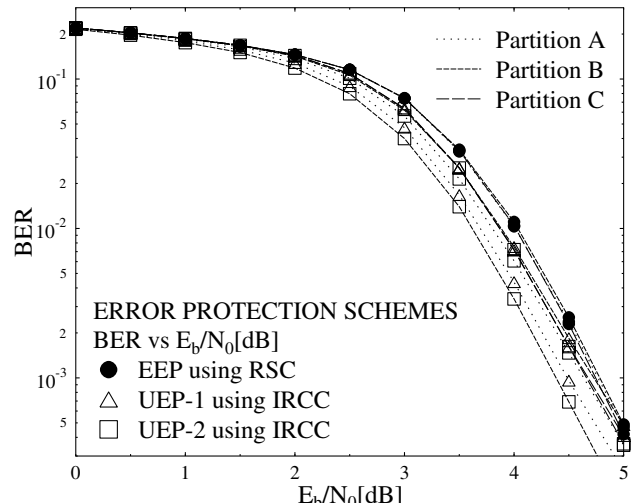

Fig. 6. BER performance of partitions $\mathrm{A}, \mathrm{B}$ and $\mathrm{C}$ of the various error protection schemes.

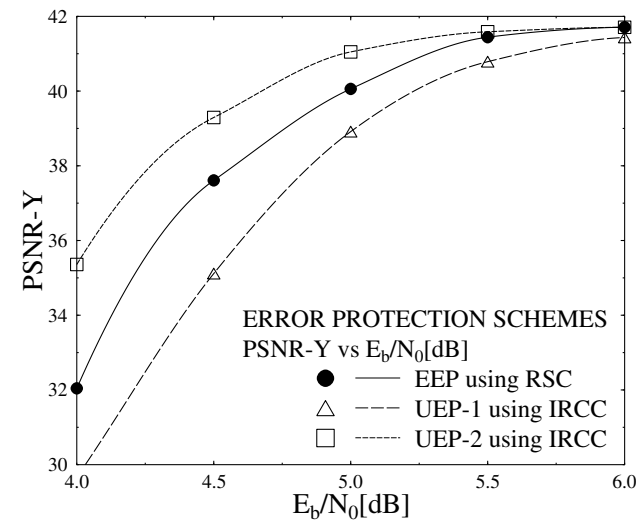

Fig. 7. PSNR-Y performance of the various error protection schemes.

along with UEP-1 and UEP-2 error protection schemes employing irregular convolutional code as outer code was recorded in Figure 8 at channel $E_{b} / N_{0}$ value of $4 d B$. In order to have a fair subjective video quality comparison, we present both the average and cumulative-error results of both the luminance and chrominance components of the 30 "Akiyo" video test sequences described in Section VII, decoded using the H.264 video codec after transmission through our proposed system for each type of setup.

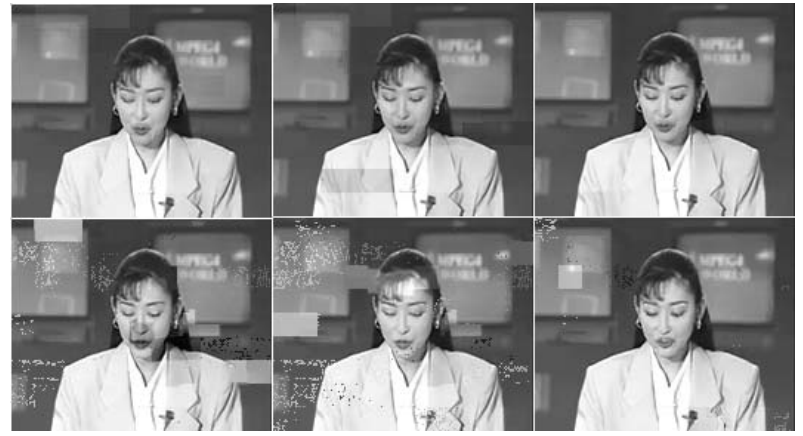

Fig. 8. Subjective video quality of the $45^{\text {th }}$ "Akiyo" video sequence frame in terms of (from top) average and cumulative-error video quality using (from left) EEP, UEP-1 and UEP-2 error protection schemes summarised in Table I at $E_{b} / N_{0}=4 d B$.

\section{CONCLUSIONS}

In this paper we proposed UEP H.264/AVC coded video transmissions using IRCC. We considered various error protection schemes designed for the transmission of H.264/AVC coded video using a serially concatenated turbo transceiver, consisting of an EXIT chartoptimised outer IRCC and an inner rate- 1 precoder. Additionally, the convergence behaviour of the system was analysed using EXIT charts. We have demonstrated that by using UEP employing an appropriate bit-rate budget allocation to different partitions of the H.264/AVC coded video based on their relative importance resulted in useful PSNR improvements for the transmitted video sequence.

\section{REFERENCES}

[1] S. X. Ng, J. Y. Chung, and L. Hanzo, "Turbo-detected unequal protection MPEG-4 wireless video telephony using multi-level coding, trellis coded modulation and space-time trellis coding," in Communications, IEE Proceedings-, vol. 152, pp. 1116-1124, Dec. 2005.

[2] L. Hanzo, C. Somerville, and J. Woodard, Voice and Audio Compression for Wireless Communications, 2nd Edition. Wiley-IEEE Press, 2007.

[3] M. Tuchler, "Design of serially concatenated systems depending on the block length," IEEE Transactions on Communications, vol. 52, pp. 209218, Feb. 2004.

[4] T. Stockhammer, M. M. Hannuksela, and T. Wiegand, "H.264/AVC in wireless environments," IEEE Transactions on Circuits and Systems for Video Technology, vol. 13, pp. 657-673, July 2003.

[5] L. Hanzo, P. Cherriman, and J. Streit, Video Compression and Communications: From Basics to H.261, H.263, H.264, MPEG2, MPEG4 for DVB and HSDPA-Style Adaptive Turbo-Transceivers. Wiley-IEEE Press, 2007

[6] A. Guyader, E. Fabre, C. Guillemot, and M. Robert, "Joint sourcechannel turbo decoding of entropy-coded sources," IEEE Journal on Selected Areas in Communications, vol. 19, pp. 1680-1696, Sept. 2001.

[7] R. G. Maunder, J. Wang, S. X. Ng, L. L. Yang, and L. Hanzo, "On the performance and complexity of irregular variable length codes for near-capacity joint source and channel coding," IEEE Transactions on Wireless Communications, vol. 7, pp. 1338-1347, Apr. 2008.

[8] L. Hanzo, P. Cherriman, and E. L. Kuan, "Interactive cellular and cordless video telephony: State-of-the-art system design principles and expected performance," Proceedings of the IEEE, vol. 88, pp. 13881413, Sept. 2000.

[9] J. Liu, G. Tu, C. Zhang, and Y. Yang, "Joint source and channel decoding for variable length encoded turbo codes," EURASIP J. Adv. Signal Process, vol. 2008, no. 1, pp. 1-10, 2008.

[10] . Nasruminallah, M. El-Hajjar, N. Othman, A. Quang, and L. Hanzo, "Over-complete mapping aided, soft-bit assisted iterative unequal error protection h.264 joint source and channel decoding," in IEEE VTC'08 (Fall), September 2008.

[11] . Nasruminallah and L. Hanzo, "Short block codes for guaranteed convergence in soft-bit assisted iterative joint source and channel decoding," IEE Electronics Letters, pp. 1315-1316, September 2008.

[12] R. Y. S. Tee, S. X. Ng, and L. Hanzo, "Precoder-aided iterative detection assisted multilevel coding and three-dimensional EXIT-chart analysis," in Wireless Communications and Networking Conference, 2006. WCNC 2006. IEEE, vol. 3, pp. 1322-1326, Apr. 2006.

[13] R. G. Maunder, J. Kliewer, S. X. Ng, J. Wang, L. L. Yang, and L. Hanzo, "Joint iterative decoding of trellis-based VQ and TCM," IEEE Transactions on Wireless Communications, vol. 6, pp. 1327-1336, Apr. 2007.

[14] M. Adrat and P. Vary, "Iterative source-channel decoding: improved system design using EXIT charts," EURASIP J. Appl. Signal Process., vol. 2005, no. 1, pp. 928-941, 2005.

[15] P. Robertson, E. Villebrun, and P. Hoeher, "A comparison of optimal and sub-optimal MAP decoding algorithms operating in the log domain," in Communications, 1995. ICC '95 Seattle, 'Gateway to Globalization', 1995 IEEE International Conference on, vol. 2, (Seattle, WA, USA), pp. 1009-1013, June 1995.

[16] S. Benedetto, D. Divsalar, G. Montorsi, and F. Pollara, "Serial concatenation of interleaved codes: performance analysis, design and iterative decoding," IEEE Transactions on Information Theory, vol. 44, pp. 909926, May 1998.

[17] M. Tuchler and J. Hagenauer, "EXIT charts of irregular codes," in Proceedings of the Conference on Information Sciences and Systems, Princeton, NJ, USA, pp. 748-753, May 2002.

[18] S. ten Brink, "Convergence behavior of iteratively decoded parallel concatenatedcodes," IEEE Transactions on Communications, vol. 49, pp. 1727-1737, Oct. 2001. 